\title{
Máxima retención de ácido ascórbico, compuestos bioactivos y capacidad antioxidante en el néctar de tumbo
}

\author{
Christian Rene Encina Zelada \\ Universidad Nacional Agraria La Molina \\ Laura Julissa Carpio Rivadeneira \\ Universidad Nacional Federico Villarreal
}

Ingeniería Industrial n² 29, 2011, ISSN 1025-9929, pp. 225-245

Recibido: 26 de marzo del 2011 / Aprobado: 16 de mayo del 2011

\begin{abstract}
REsumen: Se maximizó el ácido ascórbico del néctar de tumbo con un $\mathrm{pH}$ de 2,88; 13 grados brix, dilución pulpa:agua de 1:1 y una temperatura de pasteurización de $90^{\circ} \mathrm{C}$ aplicando los métodos Taguchi y superficie de respuesta. Se alcanzó una retención de los compuestos bioactivos del néctar en comparación con la fruta para el ácido ascórbico, carotenos totales y compuestos fenólicos del 61,81; 72,68 y 64,22\%, respectivamente; obteniéndose una capacidad antioxidante de 323,75 $\mu \mathrm{g}$ eq trolox/g (DPPH, fase hidrofílica) y de 349,91 y 471,54 $\mu \mathrm{g}$ eq trolox/g (ABTS •+, fase hidrofílica y lipofílica), respectivamente.
\end{abstract}

Palabras clave: compuestos fenólicos / métodos Taguchi / superficie de respuesta / tumbo / antioxidantes / frutas andinas / Perú

\section{Maximum retention of ascorbic acid, bioactive compounds and antioxidant capacity in banana passion fruit nectar}

ABSTRACT: Ascorbic acid was maximized in banana-passion-fruit nectar using Taguchi methods and response surface, with a $\mathrm{pH}$ of 2,88; 13 brix degrees, flesh: water dilution of $1: 1$ and a pasteurization temperature of $90{ }^{\circ} \mathrm{C}$. Nectar's bioactive compounds in comparison to the fruit decreased for ascorbic acid, carotenoids and phenolic compounds obtaining a retention of 61,$81 ; 72,68$ and $64,22 \%$ respectively, resulting in an antioxidant capacity of $323,75 \mathrm{mg}$ trolox eq/g (by DPPH in hydrophilic phase) and 349,91 and 471,54 mg trolox eq/g (by ABTS • + in hydrophilic and lipophilic phase), respectively.

Keywords: phenolic compounds / Taguchi methods / response surface / lurch / antioxidants / Andean fruits / Perú 


\section{INTRODUCCIÓN}

El tumbo (Passiflora mollisima, H.B.K., Bailey) es originario de los valles interandinos y de la zona quechua, y se encuentra en todos los Andes del Perú. Llamado anteriormente poro poro, poseía un lugar privilegiado entre las frutas de los incas (Fries 1996, citado por Arroyo 1998). También se le puede hallar en otros países de América; aunque es conocido con otras denominaciones, como "curuba" en Colombia, "parcha" en Venezuela, "granadilla cimarrona" en México y "taxo" en Ecuador.

Según Tapia (1990, citado por Arroyo 1998), el tumbo es una planta de zonas altas y frías; se desarrolla bien entre los 2000 a $3000 \mathrm{msnm}$, y se adapta al clima monzónico, con temporadas alternantes y secas.

El tumbo es una de las frutas con un alto contenido de ácido ascórbico (66,7 mg) en $100 \mathrm{~g}$ de porción comestible (Collazos et al. 1996). Generalmente se consume en estado natural; pero como posee buenas características de agradable aroma y sabor acidulado, las que son algo termosensibles, también se puede consumir en forma de jugos concentrados, jaleas, néctares, mermeladas y diversos postres (Arroyo 1998).

La producción de tumbo fresco en el Perú no es significativa, pues su cultivo no está muy difundido en nuestro país; en ese sentido, el comercio de este producto aún no se ha desarrollado, no obstante el avance de las exportaciones de las frutas denominadas "pasionarias" — correspondientes a la partida arancelaria 0810.90.10.00, donde se encuentran la granada, la granadilla, el maracuyá y otras pasifloras- alcanzado en el 2005, al situarse en 55,8 mil toneladas, de las 9 mil toneladas registradas en el 2004, y que en términos monetarios significó un notable progreso, al pasar de los US\$14 mil en el 2004 a los US\$85 mil en el 2005; pero cabe mencionar que de estas especies la principal fruta exportada fue la granada, que concentró el $86 \%$ de los volúmenes embarcados, seguida en importancia por la granadilla y el maracuyá, con participaciones de $8 \%$ y 6\%, respectivamente (Ampex 2006).

No obstante, según el Minag (2011), de la aún incipiente producción, y por ende exportación, del tumbo, hay que indicar que este es considerado una fruta de gran importancia dentro de la línea de cultivos emergentes, pronosticándosele su consolidación en la cadena productiva de nuevas plantas o especies poco conocidas en la actualidad, pero en las que se han descubierto propiedades singulares que las hacen atractivas para articular un mercado potencial interno. 
El tumbo contiene nutrientes, compuestos bioactivos como el ácido ascórbico, B-caroteno (provitamina A), compuestos fenólicos y otras vitaminas, que podrían proporcionar un efecto fisiológico beneficioso para la salud, el funcionamiento del organismo y el bienestar, mayor que el brindado por otros nutrientes más sencillos que contiene, dado que existe un efecto sinérgico entre los compuestos que presenta un alimento con estas características, pues le confiere una gran capacidad antioxidante. Por ello, desempeñan un papel esencial en la protección frente a los fenómenos de daño oxidativo y tienen efectos terapéuticos en un elevado número de patologías, incluyendo la cardiopatía isquémica, la aterosclerosis o el cáncer (Encina 2006).

Disminuir el riesgo de alteraciones cardiacas, prevenir varios tipos de cáncer, hacer más eficaz el funcionamiento del sistema inmunitario, evitar la obesidad, disminuir el deterioro por la edad, reducir el riesgo de la apoplejía, bajar la tensión arterial, ayudar a prevenir los defectos congénitos, retrasar el desarrollo de las cataratas, son algunos de los efectos beneficiosos del consumo de jugos, néctares y frutas, los que se pueden lograr con una dieta adecuada en la que se incluya la cantidad necesaria de estos productos (Ames et al. 1993).

En la sociedad actual, las frutas y sus derivados, especialmente los néctares, se han convertido en un componente imprescindible en cualquier dieta sana y equilibrada; ya que el aumento del consumo de frutas ha sido asociado a la disminución del riesgo de enfermedades degenerativas como el cáncer, las cataratas y las disfunciones del cerebro (Lampe 1999). Además, es comúnmente aceptado que las frutas, y por ende los néctares, contienen cantidades de vitaminas y provitaminas antioxidantes, fenoles y polifenoles antioxidantes —ácido ascórbico, tocoferoles, carotenoides, flavonoides, etcétera- que son beneficiosas para la salud.

Aunque en un principio se desarrollaron como consecuencia del exceso de producción de frutas, actualmente la totalidad de néctares se elabora a partir de frutas cultivadas para este fin.

En este caso en particular, queremos darle un enfoque tecnológico al obtener un néctar de tumbo, pues además de dar un valor agregado a esa materia prima, se busca difundir sus compuestos bioactivos en productos tan masivos como los néctares.

El objetivo principal de este trabajo fue el de analizar el $\mathrm{pH}$ y los grados brix en la elaboración del néctar de tumbo, la dilución pulpa:agua y la temperatura de tratamiento térmico, para conseguir los parámetros 
que maximizan la retención del ácido ascórbico y determinar el efecto sobre sus compuestos bioactivos y capacidad antioxidante.

\section{PARTE EXPERIMENTAL}

\subsection{Lugar de ejecución}

El presente trabajo de investigación se realizó en el laboratorio de Tecnología de Alimentos de la Universidad Nacional Federico Villarreal y en los laboratorios de Físico-Química, Microbiología y Planta Piloto de Alimentos, de la Facultad de Industrias Alimentarias de la Universidad Nacional Agraria La Molina.

\subsection{Materiales y reactivos}

La realización de la investigación determinó el uso de los siguientes materiales:

Muestras: Tumbo (Passiflora mollisima H.B.K. Bayley) proveniente de Ayacucho.

Reactivos: ABTS (2,2'-azino-bis(3-thylbenzthiozoline-6-sulphonic) acid) adquirido de Sigma Chemical Co. ${ }^{\circledR}$; DPPH (1,1-diphenyl-2-picrylhydrazil), de Aldrich Chemical Co. ${ }^{\circledR}$; persulfato de potasio de grado analítico de Harleco $7574^{\circledR}$; etanol absoluto y hexano de J.T. Baker ${ }^{\circledR}$; metanol de Mallinckrodt ${ }^{\circledR}$; acetona, folin-ciocalteu, carbonato de sodio anhidro al $1 \mathrm{~N}$; 2,6 diclorofenol indofenol de Ferak 33095 ${ }^{\circledR}$; ácido $1(+)$ ascórbico para análisis de Riedel-di Haën $33034^{\circledR}$; ácido metafosfórico,

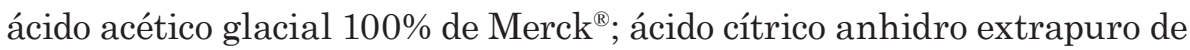
Schardau ${ }^{\circledR}$ y alcohol de $96^{\circ}$ GL.

\subsection{Métodos de análisis}

Se aplicaron los siguientes métodos de análisis:

- Análisis proximal: humedad, cenizas, lípidos, proteína total, fibra cruda, extracto libre de nitrógeno (AOAC 1990).

- Análisis fisicoquímicos: pH, acidez titulable y contenido de sólidos solubles (AOAC 1990). 
- Capacidad antioxidante medida por los métodos DPPH y ABTS (Brand-Williams et al. 1995, Re et al. 1999).

- Compuestos fenólicos totales (Swain y Hillis 1959).

- Carotenos totales (Talcott 1999), expresado como mg b-caroteno/100 g de muestra.

- Ácido ascórbico, determinado por el método de titulación con 2,6 diclorofenol indofenol (James 1995), expresado como mg ácido ascórbico/100 g muestra.

\subsection{Metodología experimental}

A continuación se describen las etapas de la metodología experimental empleada:

- Etapa 1: Caracterización de la materia prima.- Está constituida por los siguientes tipos de análisis:

- Análisis físico. Se determinaron como características físicas más resaltantes del tumbo, el tamaño, el peso (Reina 1996) y los estados de madurez (Laguado et al. 1999); los frutos se seleccionaron sobre la base de tres estados de madurez: verdes (sin olor, firme al tacto); madurez intermedia o "pintones" (predominio del color verde sobre el amarillo, desarrollo del aroma, indicios de ablandamiento); y maduros (totalmente amarillo, aroma penetrante, blando al tacto) (figura 1).

- Análisis proximal y fisicoquímico. Se trabajó con pulpa de tumbo obtenida del proceso de pulpeado y refinado, con un estado de madurez intermedio (pintón), dado que este se determinó como el apropiado para la posterior elaboración del néctar.

- Análisis químico. Se realizó la determinación del ácido ascórbico, carotenos totales, compuestos fenólicos totales y capacidad antioxidante con los métodos DPPH y ABTS.

- Análisis microbiológico. En la pulpa de tumbo, con un estado de madurez intermedio, se hicieron los análisis microbiológicos de recuento total de microorganismos aerobios y el recuento total de mohos y levaduras (I.C.M.S.F. 2000).

- Etapa 2. Determinación de los factores significativos $(p<0,05)$ que influyen en la retención del ácido ascórbico durante el proceso de elaboración del néctar de tumbo.- Se determinaron los factores que influyen significativamente en la retención del ácido ascórbico durante 
la elaboración del néctar de tumbo. Se consideraron como factores de estudio la dilución del néctar y la temperatura del tratamiento térmico, así como las características de $\mathrm{pH}$ y grados brix del néctar de tumbo. Estos factores y los niveles evaluados se muestran en la tabla 1.

\section{Tabla 1}

Factores y sus niveles considerados en el diseño experimental Taguchi $L_{9}(3)^{2}$ aplicado a la máxima retención del ácido ascórbico

\begin{tabular}{lccc}
\hline \multicolumn{1}{c}{ Factores } & Máximo & Medio & Mínimo \\
\hline F1: pH del néctar & 3,2 & 3,0 & 2,8 \\
F2: grados brix del néctar & 15,0 & 13,5 & 12,0 \\
F3: dilución de la pulpa de tumbo:agua & $1,0: 4,5$ & $1: 3,25$ & $1,0: 2,0$ \\
F4: temperatura del tratamiento térmico $\left({ }^{\circ} \mathrm{C}\right)$ & 95 & 90 & 85 \\
\hline
\end{tabular}

Elaboración propia.

- Etapa 3. Maximización de la retención de ácido ascórbico durante la elaboración del néctar de tumbo.- Se realizó mediante el método de superficie de respuesta, trabajando con los factores significativos que resultaron de la evaluación de la etapa anterior.

- Etapa 4: Caracterización del producto final.- Para examinar el producto final "néctar de tumbo" se realizaron los siguientes análisis: fisicoquímico y proximal, $\mathrm{pH}$, sólidos solubles, acidez titulable, densidad relativa, sólidos en suspensión, todos ellos necesarios para la caracterización de un néctar según la Norma Técnica Peruana (Itintec 1979); análisis químico y análisis microbiológico.

\subsection{Análisis estadístico}

Los resultados están expresados como el valor medio \pm desviación estándar de tres réplicas. En la etapa 2, para determinar los factores significativos $(p<0,05)$ se aplicó el método Taguchi (Marfil 1991, Encina 2006), el cual consistió en trabajar con un arreglo ortogonal $\mathrm{L}_{9}(3)^{2}$ que involucra el análisis de cuatro factores con tres niveles (máximo, mínimo y medio), determinando nueve tratamientos (formulaciones), los que fueron evaluados por triplicado, siendo el programa estadístico utilizado 
el Statistica â. Para la etapa 3 se aplicó la metodología de superficie de respuesta, trabajando con un arreglo factorial $3^{\mathrm{n}}$, a los factores y sus niveles seleccionados de la etapa anterior, obteniendo con ello la máxima retención de ácido ascórbico; para el tratamiento de los resultados experimentales en esta etapa se utilizó el paquete estadístico Statgraphicâ.

\section{RESULTADOS Y DISCUSIÓN}

\subsection{Caracterización de la materia prima}

\subsubsection{Análisis físico}

Para la elaboración del néctar de tumbo se trabajó la materia prima con un estado de madurez intermedio ("pintón"), tomando en cuenta lo descrito por Laguado et al. (1999) y Cheftel (1976) (mencionados por Arroyo 1998), quienes refieren que el contenido de azúcares y ácidos está estrechamente relacionado con la madurez de la materia prima, es decir que la maduración presupone un descenso de la acidez e incremento de azúcares, hasta un punto después del cual existe una degradación de compuestos bioactivos al encontrarse la materia prima en un estado de sobremadurez. Al buscar la máxima retención de ácido ascórbico trabajamos con el tumbo en estado de madurez intermedio. En la tabla 2 se muestran las características físicas del tumbo en estado de madurez intermedio.

Físicamente, Laguado et al. (1999) caracteriza el estado de madurez pintón de una fruta como aquella de color verde sobre amarillo, desarrollando el aroma, y con indicios de ablandamiento, lo cual corresponde a las características analizadas en la materia prima utilizada en el presente trabajo (figura 1). 
Figura 1

Características físicas de tres diferentes estados de madurez del tumbo

\begin{tabular}{|c|c|c|}
\hline Verdes & Olor & Hojas de plantas \\
\hline$\cdots$ & Color & Verde \\
\hline & Textura & Dura al tacto \\
\hline \multirow[t]{4}{*}{ Pintones } & & \\
\hline & Olor & Aroma en desarrollo \\
\hline & Color & Amarillo- verdosos \\
\hline & Textura & Ligero ablandamiento \\
\hline \multirow[t]{4}{*}{ Maduros } & & \\
\hline & Olor & $\begin{array}{l}\text { Fruta tropical (típica de la fruta } \\
\text { misma) }\end{array}$ \\
\hline & Color & Amarillo \\
\hline & Textura & Blando \\
\hline
\end{tabular}

Elaboración propia.

Tabla 2

Características físicas del tumbo en estado de madurez intermedio ("pintón”)

\begin{tabular}{lll}
\hline \multicolumn{2}{c}{ Características físicas } & Valor \\
\hline \multirow{2}{*}{ Tamaño } & Longitud $(\mathrm{cm})$ & $8 \pm 1$ \\
& Diámetro $(\mathrm{cm})$ & $5 \pm 1$ \\
\multirow{2}{*}{ Peso } & Fruta entera $(\mathrm{g})$ & $75 \pm 3$ \\
& Parte comestible $(\mathrm{g})$ & $47 \pm 3$ \\
& Cáscara más semillas $(\mathrm{g})$ & $28 \pm 3$ \\
\hline
\end{tabular}

Elaboración propia. 


\subsubsection{Análisis proximal y fisicoquímico}

Se observa que la humedad hallada es ligeramente superior a la reportada por Collazos et al. (1996); al mismo tiempo, es ligeramente menor que los datos reportados por Balbachas (2004), Ucharra (1985) e Izurieta et al. (1983) (mencionados por Arroyo 1998); estas diferencias de resultados pueden deberse a los distintos tipos de tumbo muestreados y también al estado de madurez de la materia prima. El contenido de cenizas hallado es parecido a los datos reportados por Collazos et al. (1996) (tabla 3).

Tabla 3

Composición proximal y fisicoquímica de la pulpa de tumbo

\begin{tabular}{lc}
\hline Parámetro & Contenido \\
\hline Humedad (\%) & $87,32 \pm 1,84$ \\
Cenizas (\%) & $0,88 \pm 0,03$ \\
Lípidos (\%) & $0,03 \pm 0,00$ \\
Proteína total (\%) & $0,92 \pm 0,08$ \\
Fibra (\%) & $0,93 \pm 0,09$ \\
Extracto libre de nitrógeno (\%) & $9,92 \pm 0,52$ \\
pH & $3,28 \pm 0,08$ \\
Acidez titulable (g de ácido cítrico/100ml) & $4,1 \pm 0,12$ \\
Sólidos solubles ( ${ }^{\circ}$ Brix) & $12 \pm 0,5$ \\
\hline
\end{tabular}

Elaboración propia.

El contenido de proteínas y fibra es ligeramente menor que el reportado por Collazos (1996), Balbachas (2004) y Nina (1993), mencionados por Arroyo (1998). El bajo contenido de proteínas y grasa según Davies y Albrigo (1994, mencionados por Encina 2006), se debe a las características cítricas del alimento. El contenido de ácido ascórbico es ligeramente menor que el reportado por Collazos et al. (1996), pequeñas diferencias que se pueden deber a que las muestras fueron obtenidas en distintas estaciones, como lo señalan Davies y Albrigo (1994, mencionados por Encina 2006).

\subsubsection{Análisis químico de compuestos bioactivos}

Se determinaron como compuestos bioactivos en la pulpa de tumbo su 
contenido de ácido ascórbico, carotenos totales, compuestos fenólicos, los responsables — entre otros compuestos - de su actividad antioxidante, determinada por los métodos DPPH y ABTS, resultados que se muestran en la tabla 4.

Tabla 4

Análisis de los compuestos bioactivos y capacidad antioxidante del tumbo

\begin{tabular}{|c|c|c|}
\hline \multicolumn{2}{|l|}{ Componente } & Contenido \\
\hline \multicolumn{2}{|l|}{ Ácido ascórbico (mg / 100 g) } & $61,80 \pm 3,58$ \\
\hline \multicolumn{2}{|c|}{ Carotenos totales (mg de $\beta$-caroteno $/ 100 \mathrm{~g}$ ) } & $1,83 \pm 0,03$ \\
\hline \multicolumn{2}{|c|}{ Compuestos fenólicos (mg ácido clorogénico/100 g) } & $190,32 \pm 2,14$ \\
\hline \multirow{3}{*}{$\begin{array}{l}\text { Capacidad antioxidante } \\
(\mu \mathrm{g} \text { eq trolox/g) }\end{array}$} & Hidrofílica & $494,32 \pm 12,42$ \\
\hline & Hidrofílica & $578,59 \pm 19,26$ \\
\hline & Lipofílica & $679,14 \pm 17,32$ \\
\hline
\end{tabular}

Elaboración propia.

Los resultados promedios de carotenoides totales $(0,83 \mathrm{mg}$ de B-caroteno/100g, lo que equivale a 3050 UI de vitamina A) obtenidos para el tumbo con estado de madurez intermedia utilizado como materia prima para la elaboración del néctar presenta un nivel bajo de B-caroteno, pero tal como menciona Rodríguez-Amaya (1999, citado por Encina 2006), aquellos con un nivel bajo de B-caroteno (12 a $23 \mu \mathrm{g} / \mathrm{g}$ ), en la mayoría de las frutas y vegetales que contienen carotenoides, la maduración se ve acompañada por un aumento de la biosíntesis de carotenoides, como en el damasco, el mango, el melón, la papaya, etcétera.

Respecto al contenido de compuestos fenólicos, para el estado de madurez evaluado un 190,32 mg ácido clorogénico/100 g, el cual es un valor relativamente alto respecto a los valores mencionados por Lister y Podivinsky (1998), los que presentaron el contenido de compuestos fenólicos totales de algunos vegetales en base húmeda, entre los cuales están la lechuga roja (182 mg ácido clorogénico/100 g), el brócoli (83,1 mg ácido clorogénico/100 g), la zanahoria (40,2 mg ácido clorogénico/100 g), la cebolla (66,8 mg ácido clorogénico/100 g), la coliflor (35 mg ácido clorogénico/100 g), la lechuga-corazón (24,4 mg ácido clorogénico/100 g) y el tomate (28,8 mg ácido clorogénico/100 g).

Se determinó la capacidad antioxidante del tumbo, el resultado promedio para un estado de madurez intermedio fue de 494,32 $\mu \mathrm{g}$ eq 
trolox/g según el método del DPPH, 578,59 (parte hidrofílica) y 679,14 (parte lipofílica) $\mu$ g eq trolox/g según el método del ABTS. La capacidad antioxidante de un alimento depende de la naturaleza y concentración de los antioxidantes naturales presentes en él. El tomate tiene el menor valor de capacidad antioxidante, a pesar de tener grandes cantidades de licopeno, probablemente porque el licopeno y los carotenoides en general, son eficientes en la extinción (quenching) de singletes de oxígeno, y no tanto en el atrapamiento de radicales peroxílicos (Pineda et al. 1999, citado por Encina 2006).

Se debe mencionar que para evaluar la capacidad antioxidante mediante el método del DPPH se utilizó como solvente el metanol, es decir se cuantificó la capacidad antioxidante de compuestos hidrófilos (ácido ascórbico y compuestos fenólicos), mientras que para la evaluación de la capacidad antioxidante mediante el método del ABTS se utilizó como solvente el metanol inicialmente, y luego una mezcla de isopropanol/ hexano, es decir se cuantificó la capacidad antioxidante de compuestos hidrófilos (ácido ascórbico y compuestos fenólicos) y compuestos lipófilos (carotenoides), razón por la cual en el segundo caso se obtuvo una mayor capacidad antioxidante.

\subsubsection{Análisis microbiológico}

Como se observa en la tabla 5, la pulpa de tumbo contaba con un bajo contenido de unidades formadoras de colonia (u.f.c.), estos valores son relativamente bajos, especialmente los de hongos y levaduras, debido a que el tumbo es una fruta con una cubierta gruesa. El bajo contenido de hongos y levaduras (100 u.f.c./g) también se puede deber al bajo pH que contiene la pulpa de tumbo $(3,28)$, ya que I.C.M.S.F. (2000) señala que todos los hongos y levaduras crecen bien a valores de $\mathrm{pH} 5$ o mayores.

Tabla 5

Carga microbiana de la pulpa de tumbo (Passiflora mollissima)

\begin{tabular}{lc}
\hline Indicador & u.f.c./g \\
\hline Recuento de aerobios mesófilos viables & 400 \\
Recuento de hongos y levaduras & 100 \\
\hline
\end{tabular}

Elaboración propia. 
3.2 Determinación de los factores $(p<0,05)$ que influyen en la retención del ácido ascórbico del néctar de tumbo

Se trabajó con cuatro factores durante la elaboración del néctar, y sus niveles fueron hallados a través de pruebas preliminares, de esta forma se determinaron los grados brix del néctar como un mínimo de 12 y un máximo de 15 , un $\mathrm{pH}$ un mínimo de 2,8 y máximo de 3,2 ; valor mínimo de dilución de pulpa:agua de 1:2 y máximo de 1:4,5; y para el tratamiento térmico del néctar una temperatura mínima de $85^{\circ} \mathrm{C}$ y máxima de $95^{\circ} \mathrm{C}$, teniendo como la variable respuesta de estos análisis la determinación del contenido de ácido ascórbico en cada uno de los tratamientos.

Según el diseño experimental Taguchi $\mathrm{L}_{9}(3)^{2}$ se realizaron nueve tratamientos por triplicado, de los cuales para cada tratamiento, y con un nivel de significancia de $95 \%(\mathrm{p}<0,05)$, se determinó la cantidad de ácido ascórbico retenido. En la tabla 6 se muestran tales tratamientos con los valores hallados de ácido ascórbico. La selección de la formulación óptima del néctar de tumbo se basó en el criterio "mayor es mejor" (a mayor cantidad de ácido ascórbico, el producto final es mejor) según uno de los fundamentos del método de Taguchi. Para esto, una medida estadística de la performance es la razón señal-ruido (S/R) o "ETA", que es usada para evaluar la calidad de un producto, una mayor performance es medida por una razón mayor de ETA, lo que implica menor pérdida (Encina 2006).

Tabla 6

Tratamientos según el diseño experimental de Taguchi $L_{9}(3)^{2}$ y sus resultados para el contenido de ácido ascórbico

\begin{tabular}{|c|c|c|c|c|c|}
\hline \multicolumn{5}{|c|}{ Factores de control } & \multirow{2}{*}{$\begin{array}{l}\text { Ácido ascórbico } \\
(\mathrm{mg} / 100 \mathrm{ml})\end{array}$} \\
\hline $\begin{array}{l}\text { Trata- } \\
\text { miento }\end{array}$ & $\begin{array}{l}\text { pH del } \\
\text { néctar }\end{array}$ & $\begin{array}{l}\text { Grados brix } \\
\text { del néctar }\end{array}$ & $\begin{array}{l}\text { Dilución } \\
\text { pulpa: } \mathrm{H}_{2} \mathrm{O}\end{array}$ & $\begin{array}{l}\text { Temperatura de } \\
\text { pasteurización }\end{array}$ & \\
\hline 1 & 2,8 & 12 & $1: 2,0$ & 85 & $24,35 \pm 0,65$ \\
\hline 2 & 2,8 & 13,5 & $1: 3,25$ & 90 & $21,87 \pm 2,21$ \\
\hline 3 & 2,8 & 15 & $1: 4,5$ & 95 & $11,73 \pm 1,56$ \\
\hline 4 & 3 & 12 & $1: 3,25$ & 95 & $13,70 \pm 1,01$ \\
\hline 5 & 3 & 13,5 & $1: 4,5$ & 85 & $10,60 \pm \mathbf{0 , 5 8}$ \\
\hline 6 & 3 & 15 & $1: 2,0$ & 90 & $21,99 \pm 2,40$ \\
\hline 7 & 3,2 & 12 & $1: 4,5$ & 90 & $11,19 \pm 0,09$ \\
\hline 8 & 3,2 & 13,5 & $1: 2,0$ & 95 & $21,83 \pm 1,58$ \\
\hline 9 & 3,2 & 15 & $1: 3,25$ & 85 & $16,24 \pm 1,42$ \\
\hline
\end{tabular}

Elaboración propia. 
Figura 2

Valores señal/ruido $(S / R)$ de los factores evaluados para maximizar la retención del ácido ascórbico aplicando el diseño ortogonal Taguchi L9(34)

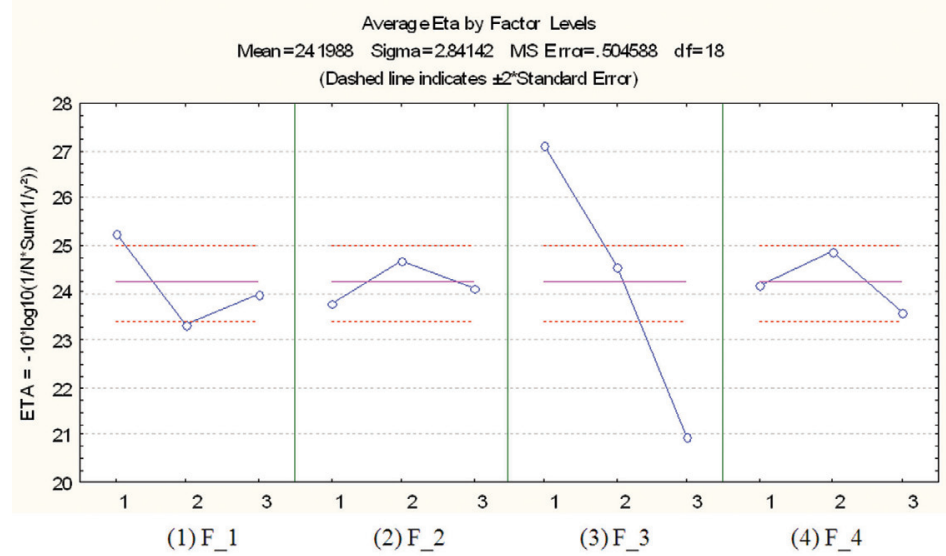

Donde:

F_1: pH del néctar;

F_2: grados brix del néctar;

F_3: dilución agua: pulpa, y

F_4: temperatura de tratamiento térmico

Elaboración propia.

El factor significativo, según lo mostrado en la figura 2, fue la dilución de pulpa:agua, tuvo el más alto valor de ETA significativo, correspondiendo el menor valor de ETA al nivel menor (1:2); el pH del néctar, tuvo el segundo mayor valor de ETA, y aunque no fue significativo, nos indicaba que para obtener el mayor contenido de ácido ascórbico se debía trabajar con valores de $\mathrm{pH}$ en el nivel menor $(2,8)$, por lo que se eligieron esos dos factores con sus respectivos niveles como variables para la maximización posterior (Fennema 2000, citado por Encina 2006), indica que el $\mathrm{pH}$ ejerce una influencia en cualquier circunstancia en la degradación del ácido ascórbico. Los factores grados brix del néctar y temperatura de tratamiento térmico no fueron significativos estadísticamente, y además se logra maximizar el valor ETA en ambos casos con los valores medios (13,5 grados brix y $90^{\circ} \mathrm{C}$, respectivamente), por lo que fueron elegidos como factores constantes para la maximización posterior. En la tabla 7 se presentan los valores óptimos de cada factor para la máxima retención de ácido ascórbico en la elaboración de néctar de tumbo, según Taguchi. 
Tabla 7

Valores óptimos de cada factor para la elaboración de néctar de tumbo según Taguchi

\begin{tabular}{lcc}
\hline Factores & Parámetro & Nivel seleccionado \\
\hline pH del néctar & 2,8 & 1 \\
Grados brix del néctar & 13,5 & 2 \\
Dilución pulpa:agua & $1: 2$ & 1 \\
Temperatura de tratamiento térmico & 90 & 2 \\
\hline
\end{tabular}

Elaboración propia.

\subsection{Maximización de la retención de ácido ascórbico durante la} elaboración del néctar de tumbo

En la tabla 8 se presentan los factores y los niveles ensayados para la maximización del producto mediante el método de superficie de respuesta, los que se sometieron a la cuantificación del ácido ascórbico después de su procesamiento, donde también se observa que los grados brix del néctar y la temperatura de tratamiento térmico son factores óptimos constantes establecidos por el método de Taguchi aplicado en la etapa anterior.

Tabla 8

Valores de cada factor que maximizan la retención del ácido ascórbico obtenidos mediante superficie de respuesta

\begin{tabular}{|c|c|c|c|}
\hline Factor & Mínimo & Medio & Máximo \\
\hline $\mathrm{pH}$ del néctar & 2,5 & 2,8 & 3,1 \\
\hline Dilución agua:pulpa & $1: 1$ & $1: 2$ & $1: 3$ \\
\hline
\end{tabular}

Elaboración propia.

Después de realizadas las nueve combinaciones resultantes de los factores y sus niveles, presentados en la tabla 8, se efectuó la cuantificación del ácido ascórbico de cada uno de los tratamientos. En la figura 3 se muestran los resultados de concentración de ácido ascórbico para cada tratamiento. Estos valores fueron evaluados mediante el análisis estadístico de superficie de respuesta para la variable respuesta $\mathrm{mg}$ de 
ácido ascórbico/100g en la muestra, y para los factores en estudio $\mathrm{pH}$ del néctar y dilución pulpa:agua en el néctar de tumbo.

Los datos valores que maximizan la retención de ácido ascórbico según el método de superficie de respuesta fueron para el factor $\mathrm{A}$ ( $\mathrm{pH}$ del néctar) el nivel medio 2,88; y para el factor $\mathrm{B}$ (dilución pulpa:agua) el nivel bajo 1:1; así, a menor dilución para un néctar este va a tener mayor contenido de nutrientes, dado que se está diluyendo menos, es decir, se agrega menor contenido de agua.

Figura 3

Superficie de respuesta y sus curvas de nivel para la mayor retención de ácido ascórbico
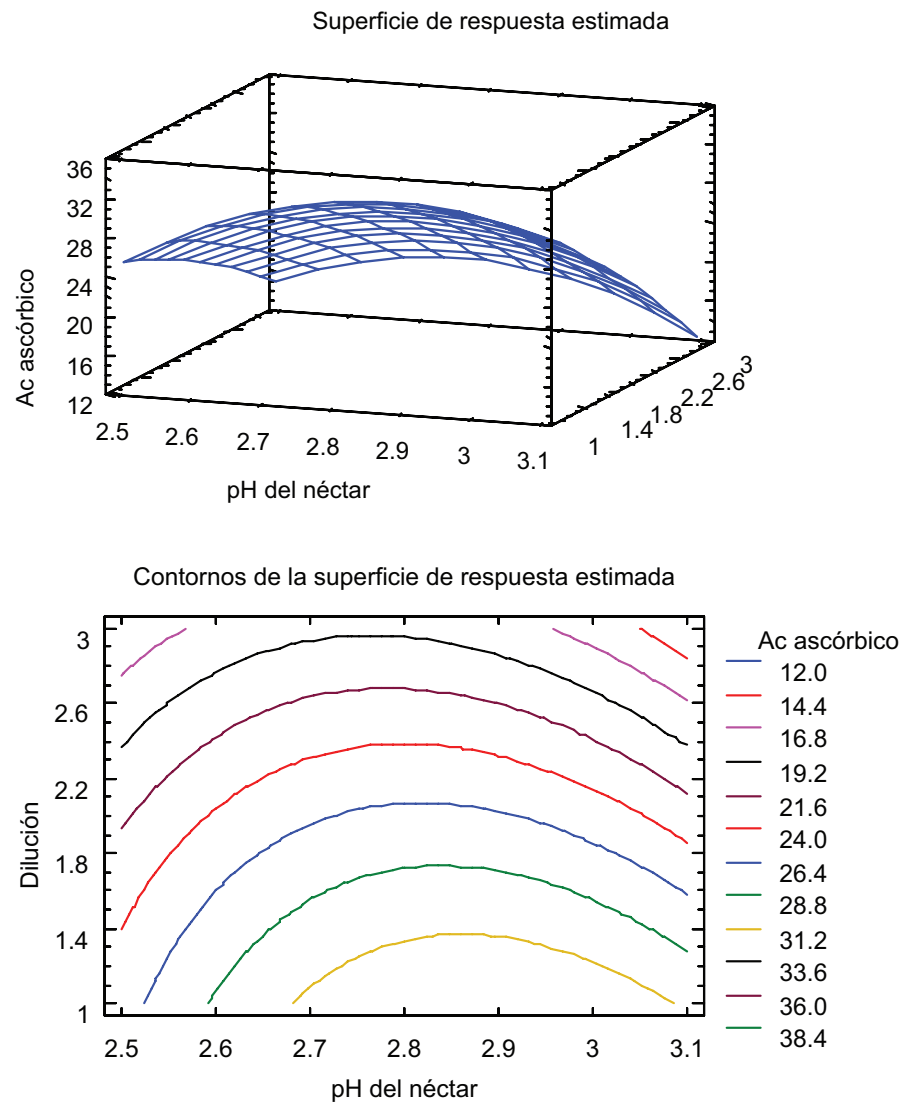

Elaboración propia. 


\subsection{Caracterización del producto final}

\subsubsection{Análisis proximal y fisicoquímico}

En la tabla 10 se muestran los resultados del análisis químico proximal del néctar de tumbo. Los valores hallados de humedad, cenizas, proteínas y fibra son: $89,32 \% ; 0,66 \% ; 0,59 \%$ y $0,69 \%$, respectivamente; los que resultan ser valores ligeramente superiores a los reportados por Quinto (2000), quien trabajó como materia prima el maracuyá (fruta cítrica con características similares a las del tumbo y de la misma familia Passifloraceae) y encontró en la elaboración de un néctar de maracuyá enriquecido con maca contenidos de $82,04 \% ; 0,46 \% ; 0,29 \%$ y $0,15 \%$, respectivamente; estas diferencias pueden deberse a que dicho autor elaboró un néctar adicionándole maca a la formulación. Asimismo, puede deberse a la dilución de pulpa:agua, ya que en el caso del néctar de maracuyá fue de 1:0,35 y para el caso del tumbo se trabajó con una dilución de 1:1.

Tabla 10

Composición química del néctar de tumbo (Passiflora mollissima)

\begin{tabular}{lc}
\hline \multicolumn{1}{c}{ Parámetro } & Contenido \\
\hline Humedad (\%) & $89,32 \pm 3,23$ \\
Cenizas (\%) & $0,66 \pm 0,02$ \\
Lípidos (\%) & $0,02 \pm 0,00$ \\
Proteína total (\%) & $0,59 \pm 0,04$ \\
Fibra (\%) & $0,69 \pm 0,04$ \\
Extracto libre de nitrógeno (\%) & $8,72 \pm 0,42$ \\
Ácido ascórbico (mg/100 ml) & $38,20 \pm 2,56$ \\
Carotenos totales (mg de & $1,33 \pm 0,03$ \\
$\beta$-caroteno/100 ml) & $2,9 \pm 0,02$ \\
pH & $13,5 \pm 0,5$ \\
Sólidos solubles ( ${ }^{\circ B i x)}$ & $1,5 \pm 0,05$ \\
Acidez titulable (g de ácido & $2,61 \pm 0,05$ \\
cítrico/100ml) & $0,33 \pm 0,01$ \\
Densidad relativa & \\
Sólidos en suspensión (v/v) & \\
\hline
\end{tabular}

Elaboración propia. 
El valor de la densidad relativa resultó 2,61 para el néctar de tumbo, según la Norma Técnica Peruana 203.011 (Indecopi 1979); para el néctar de maracuyá la densidad relativa es de un mínimo de 1,040, ya que la densidad es la masa que corresponde a un volumen, lo que permite concluir que la diferencia en valores de la densidad relativa puede deberse a la dilución de pulpa:agua, pues en este caso se elaboró el néctar con la mínima dilución (1:1).

Según la Norma Técnica 203.011 (Itintec 1979), el néctar de maracuyá tiene un pH máximo final de 3,5 y según Quinto (2000) un valor de 3,75 ; en nuestro caso resultó un $\mathrm{pH}$ final de 2,9. Esta desigualdad puede deberse a que las materias primas utilizadas son diferentes (tumbo y maracuyá), como también al estado de madurez de las muestras.

Se encontró una notable diferencia de valores con respecto a la acidez titulable del néctar de maracuyá (Itintec 1979) y del néctar de tumbo, ya que en este caso resultó $1,5 \mathrm{~g}$ de ácido cítrico/100 $\mathrm{ml}$ y según la norma técnica es de un valor de $0,8 \mathrm{~g}$ de ácido cítrico $/ 100 \mathrm{ml}$, pudiendo deberse principalmente a que la dilución que se utilizó en este caso para la elaboración del néctar de tumbo fue la mínima.

\subsubsection{Análisis químico de compuestos bioactivos}

En la tabla 11 se observa el contenido de ácido ascórbico, carotenos totales, compuestos fenólicos y la capacidad antioxidante determinada por los métodos DPPH y ABTS; en términos generales, podemos apreciar que la elaboración del néctar de tumbo genera, en todos los casos, pérdidas de los compuestos bioactivos analizados, y por ende disminución de su capacidad antioxidante, que es resultado no solo de las variables tecnológicas aplicadas para la obtención del néctar, como son la temperatura de tratamiento térmico o la estandarización del $\mathrm{pH}$ y grados brix, sino también la dilución con agua y pulpa, que tiene como finalidad hacer sensorialmente agradable el néctar, en especial de aquellas frutas ácidas cuyo consumo se realiza diluyendo varias veces parte de la pulpa. 
Tabla 11

Análisis de los compuestos bioactivos en el néctar de tumbo

\begin{tabular}{|c|c|c|c|c|}
\hline \multicolumn{3}{|l|}{ Componente } & Contenido & Retención \% \\
\hline \multicolumn{3}{|c|}{ Ácido ascórbico (mg/100 g) } & $38,20 \pm 2,56$ & 61,81 \\
\hline \multicolumn{3}{|c|}{ Carotenos totales (mg de $\beta$-caroteno/100g) } & $1,33 \pm 0,03$ & 72,68 \\
\hline \multicolumn{3}{|c|}{ Compuestos fenólicos (mg ácido clorogénico/100 g) } & $122,24 \pm 2,02$ & 64,22 \\
\hline \multirow{3}{*}{$\begin{array}{l}\text { Capacidad antioxi- } \\
\text { dante } \\
(\mu \mathrm{g} \text { eq trolox/g) }\end{array}$} & $\mathrm{DPPH}$ & Hidrofílica & $323,75 \pm 9,24$ & 65,49 \\
\hline & \multirow{2}{*}{ ABTS } & Hidrofílica & $349,91 \pm 12,66$ & 60,48 \\
\hline & & Lipofílica & $471,54 \pm 11,27$ & 69,43 \\
\hline
\end{tabular}

Elaboración propia.

Chen et al. (1995) y Rodríguez-Amaya (1999) (mencionados por Encina 2006), estudiaron el efecto de diversos métodos de procesamiento sobre el contenido de a y B-caroteno en el jugo de zanahoria. La más alta destrucción de los carotenos fue en retorta fija a $121^{\circ} \mathrm{C}$ durante 30 minutos, y la menor con la pasteurización a $105^{\circ} \mathrm{C}$ durante 25 segundos del jugo acidificado, utilizando un sistema de laboratorio. La disminución de los compuestos bioactivos en el néctar de tumbo se puede deber al efecto que tuvo el tratamiento térmico sobre los compuestos hidrofílicos (ácido ascórbico y compuestos fenólicos), así como sobre los compuestos lipofílicos (carotenoides) presentes en el producto, los cuales siguen actuando sinérgicamente, pero con una disminución de su capacidad antioxidante respecto a la materia prima sin procesar.

Existen estudios de determinación de la capacidad antioxidante en bebidas mediante la metodología del DPPH, obteniéndose valores en general que disminuyeron al realizarse el procesamiento de las frutas para obtener dichas bebidas, las que fueron sometidas también a tratamiento térmico durante su elaboración, referencias mencionadas en el estudio realizado por Murillo (2005, citado por Encina 2006).

Según Aranceta y Pérez-Rodrigo (2006), la capacidad antioxidante determinada por el método TEAC (trolox) de diferentes cultivos de bayas oscila entre 24,6 y $39 \mathrm{mmol}$ trolox/l, pero el tratamiento para obtener zumos a escala industrial es responsable de la pérdida de antioxidantes durante el procesado, disminuyendo entre $20 \%$ y $40 \%$, valores en los que se encuentra nuestro néctar de tumbo. 


\subsubsection{Análisis microbiológico}

En la tabla 12 se muestran los resultados del análisis microbiológico del néctar de tumbo. No se detectaron colonias de microorganismos aerobios mesófilos viables ni de hongos y levaduras, de acuerdo con los valores reportados por el Ministerio de Salud (Resolución N 7992 1991); se podría concluir que el néctar de tumbo elaborado cuenta con las condiciones microbiológicas apropiadas para su consumo.

Tabla 12

Carga microbiana del néctar de tumbo (Passiflora mollisima)

\begin{tabular}{lc}
\hline \multicolumn{1}{c}{ Indicador } & u.f.c./ml \\
\hline Recuento de aerobios mesófilos viables & $<10$ \\
Recuento de hongos y levaduras & $<10$ \\
\hline
\end{tabular}

Elaboración propia.

\section{CONCLUSIONES}

- Los parámetros que maximizaron la retención de ácido ascórbico en la elaboración del néctar de tumbo aplicando los métodos de Taguchi y Superficie de Respuesta fueron de 2,9 para el $\mathrm{pH}$ del néctar, 13 grados brix del néctar, una dilución pulpa:agua de 1:1 y una temperatura pasteurización de $90^{\circ} \mathrm{C}$.

- Los compuestos bioactivos del néctar de tumbo se vieron reducidos en comparación con el fruto inicial para el ácido ascórbico, carotenos totales y compuestos fenólicos en $61,81 \% ; 72,68 \%$ y $64,22 \%$, respectivamente, obteniéndose en ella una capacidad antioxidante en la fase hidrofílica determinada por el reactivo DPPH de 323,75 $\mu \mathrm{g}$ eq trolox/g y una en la fase hidrofílica y lipofílica determinada por el radical $\mathrm{ABTS}^{++}$de 349,91 y 471,54 $\mu \mathrm{g}$ eq trolox/g, respectivamente. Estos compuestos bioactivos y su capacidad antioxidante estudiados en otras investigaciones, demuestran que podrían estar relacionados con una disminución del riesgo de enfermedades degenerativas como cáncer, cataratas o disfunciones del cerebro. 


\section{BIBLIOGRAFÍA}

Ames, B. N.; Shigenaga, M. K. y T. M. Hagen (1993). "Oxidants, antioxidants, and the degenerative diseases of aging". Procedings of National Academy of Science. Vol. 90.

Asociación Macrorregional de Productores para la Exportación (Ampex) (2006). "Perfil de mercado de la granada fresca (Granatum L. de Punica)". Chiclayo. <www.ampex.com.pe/down_file.php?f=perfilgranada.pdf\&ruta=perfi>. [Consulta: 4 de febrero del 2009.]

AOAC (1990). Official methods of analysis, 15. a edición. Washington D.C.: Association of Official Analytical Chemists.

Aranceta, J. y C. Pérez-Rodrigo (2006). Frutas, verduras y salud. Barcelona: Masson.

Arroyo, E. (1998). "Néctar de tumbo". Monografía para optar al título de ingeniero alimentario. Lima: Universidad Nacional Federico Villarreal.

Brand-Williams, W.; Cuvelier, M. y C. Berset (1995). "Use of free radical method to evaluate antioxidant activity". Lebensmittel-Wissenschaft \& Technologie. Vol. 28. Zurich.

Collazos, C. et al. (1996). Tabla peruana de composición de alimentos.

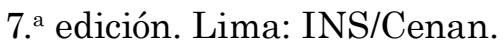

Encina, Ch. (2006). "Influencia en el descerado y composición del almíbar en la optimización del tratamiento térmico de la conserva de aguaymanto (Physalis peruviana, Linnaeeus, 1753)". Tesis para optar al grado de Magíster Scientiae. Lima: Universidad Nacional Agraria La Molina.

I.C.M.S.F. (2000). Microorganismos de los alimentos, su significado y métodos de numeración. 2. ${ }^{a}$ edición. Tomo II. Zaragoza: Acribia.

Itintec (1979). "Néctar de maracuyá". Norma Técnica No 203-011. Documento de Trabajo. Lima: Indecopi.

James, C. (1995). Analytical chemistry of foods. Londres: Blackie Academic \& Professional.

Laguado, N. et al. (1999). "Crecimiento de frutos de guayabo (Psidium guajava L.) del tipo criolla roja. Etapa I”. Revista de la Facultad de Agronomía (LUZ). Vol. 16. Maracaibo. 
Lampe, J. W. (1999). "Health effects of vegetables and fruits: assesing the mechanisms of action in human experiments studies". American Journal of Clinical Nutrition. Vol. 70.

Lister, C. y E. Podivinsky (1998). "Antioxidant in New Zealand grown fruit and vegetables". Polyphenols Communications. Vol. 98.

Marfil, R. (1991). "Una herramienta para el mejoramiento de la calidad". Tecnología de Alimentos. Vol. 25, núm. 5.

Minag (2011). "Líneas de cultivo emergente: Frutas". Lima. <http:// www.minag.gob.pe/lineas-de-cultivos-emergentes/frutas.html>.

Ministerio de Salud de Colombia (21 de junio de 1991). Resolución $\mathrm{N}^{\circ}$ 7992. "Elaboración, conservación y comercialización de jugos, concentrados, néctares, pulpas, pulpas edulcoradas y refrescos de frutas". Bogotá.

Quinto, R. (2000). "Elaboración de néctar de maracuyá enriquecido con maca". Tesis para optar al título de ingeniero alimentario. Lima: Universidad Nacional Federico Villarreal.

Re, R. et al. (1999). "Antioxidant activity applying an improved ABTS radical cation decolorization assay". Free Radical Biology and Medicine. Vol. 26.

Reina, C. et al. (1996). "Manejo poscosecha y evaluación de calidad para la guanábana (Annona maricata l.) que se comercializa en la ciudad de Neiva". Neiva: Universidad Surcolombiana. <http://www. agronet.gov.co/www/docs_si2/Manejo\%20poscosecha\%20y\%20 evaluacion $\% 20 \mathrm{de} \% 20 \mathrm{la} \% 20$ calidad $\% 20 \mathrm{en} \% 20$ Curuba.pdf $>$. [Consulta: 4 de febrero del 2009.]

Swain, T. y W. E. Hillis (1959). "The phenolic constituents of Prunus domesticus 1.- quantitative analysis of phenolic constituents". Journal of the Science of Food and Agriculture. Vol. 10.

Talcott, S. y L. Howard (1999). "Phenolic autoxidation is responsible for color degradation in processed carrot puree". Journal of Agricultural and Food Chemistry. Vol. 47. 